\title{
OPEN Publisher Correction: Spatial distribution of freshwater crustaceans in Antarctic and Subantarctic lakes
}

Angie Díaz, Claudia S. Maturana, Luz Boyero $\mathbb{D}$, Patricio De Los Ríos Escalante $\mathbb{D}$, Alan M. Tonin $(\mathbb{D}$ \& Francisco Correa-Araneda

Correction to: Scientific Reports https://doi.org/10.1038/s41598-019-44290-4, published online 28 May 2019

This Article contains errors in Table 1. In column 17 'Ref., all instances of ' 1 ' should read ' 10 '. In addition, all instances of ' 2 ' should read ' 6 '.

As a result, in the legend for Table 1,

'Presence/absence matrix of crustacean taxa in lakes of each study region based on Pugh et al. 2002 (1) and Dartnall et al. 2017 (2)'

should read:

'Presence/absence matrix of crustacean taxa in lakes of each study region based on Pugh et al. 2002 (10) and Dartnall et al. 2017 (6)'

The correct Table 1 appears below. 


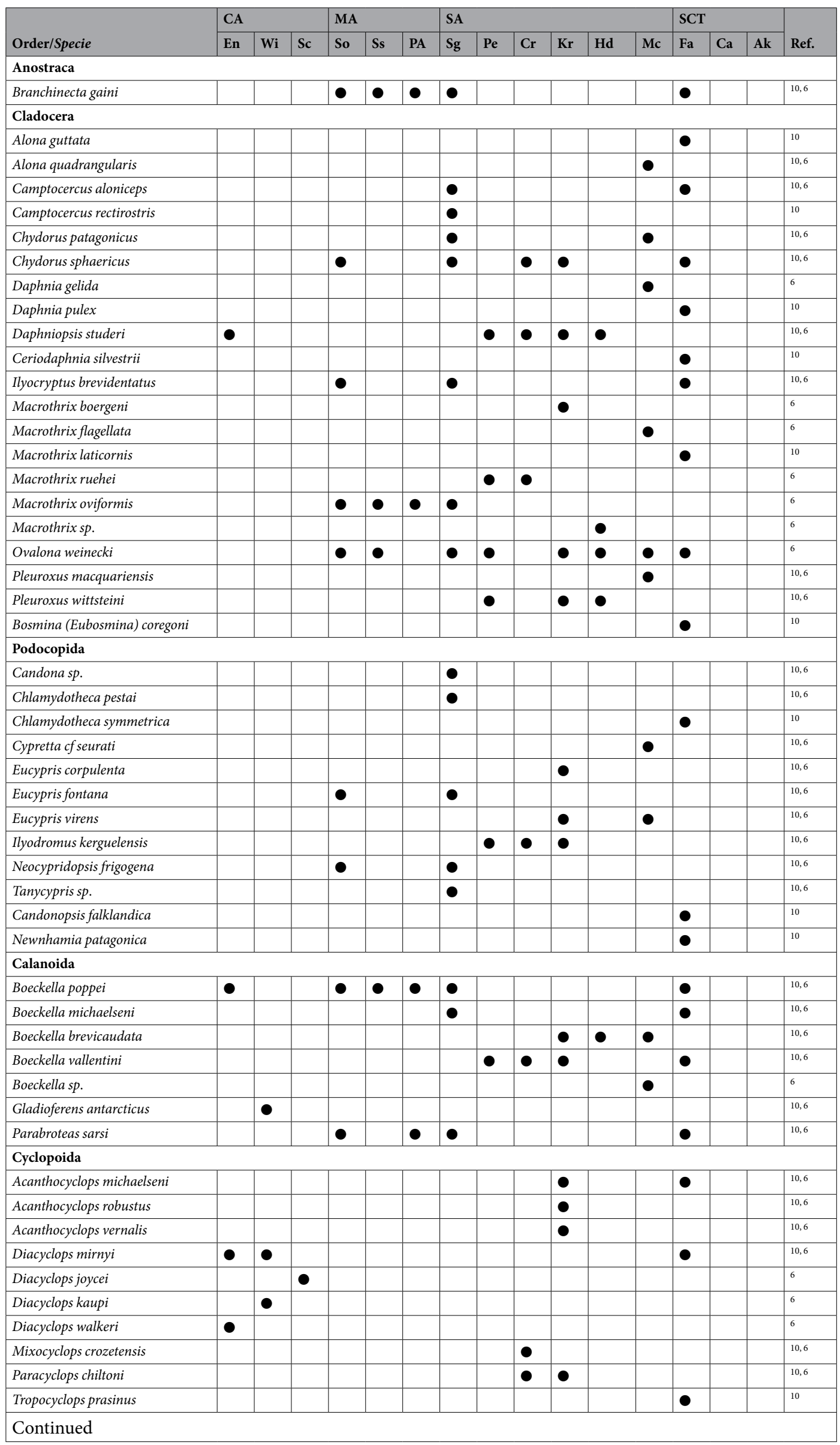




\begin{tabular}{|c|c|c|c|c|c|c|c|c|c|c|c|c|c|c|c|c|}
\hline \multirow[b]{2}{*}{ Order/Specie } & \multicolumn{3}{|l|}{ CA } & \multicolumn{3}{|c|}{ MA } & \multicolumn{6}{|l|}{ SA } & \multicolumn{3}{|c|}{ SCT } & \multirow[b]{2}{*}{ Ref. } \\
\hline & En & $\mathbf{W i}$ & Sc & So & Ss & PA & Sg & $\mathrm{Pe}$ & $\mathbf{C r}$ & $\mathbf{K r}$ & Hd & Mc & $\mathbf{F a}$ & $\mathrm{Ca}$ & Ak & \\
\hline \multicolumn{17}{|l|}{ Harpacticoida } \\
\hline Antarctobiotus koenigi & & & & & & & $\bullet$ & & & & & & & & & 10,6 \\
\hline Antarctobiotus robustus & & & & & & & & & $\bullet$ & $\bullet$ & & & & & & 10,6 \\
\hline Epactophanes richardi & & & & & & & & $\bullet$ & $\bullet$ & $\bullet$ & $\bullet$ & & & & & 10,6 \\
\hline Marionobiotus jeanneli & & & & & & & & $\bullet$ & & $\bullet$ & & $\bullet$ & & & & 10,6 \\
\hline Marionobiotus sp. & & & & & & & & & & & & $\bullet$ & & & & 6 \\
\hline Tigriopus angulatus & & & & & & & & $\bullet$ & $\bullet$ & $\bullet$ & & $\bullet$ & & & & 10,6 \\
\hline Attheyella (D.) trigonura & & & & & & & & & & & & & $\bullet$ & & & 10 \\
\hline \multicolumn{17}{|l|}{ Amphipoda } \\
\hline Kergueleniola macra & & & & & & & & & & $\bullet$ & & & & & & 10,6 \\
\hline Pseudingolfiella possessionis & & & & & & & & & $\bullet$ & & & & & & & 6 \\
\hline Chiltonia mihiwaka & & & & & & & & & & & & & & $\bullet$ & - & 10 \\
\hline Hyalella curvispina & & & & & & & & & & & & & $\bullet$ & & & 10 \\
\hline Hyalella neonoma & & & & & & & & & & & & & $\bullet$ & & & 10 \\
\hline Falklandella obtusa & & & & & & & & & & & & & $\bullet$ & & & 10 \\
\hline Praefalklandella cuspidatus & & & & & & & & & & & & & $\bullet$ & & & 10 \\
\hline \multicolumn{17}{|l|}{ Isopoda } \\
\hline Lais sp. & & & & & & & & & & & & $\bullet$ & & & & 6 \\
\hline No total species & 4 & 3 & 1 & 9 & 4 & 4 & 17 & 9 & 11 & 19 & 6 & 14 & 25 & 1 & 1 & \\
\hline No species by zones & \multicolumn{3}{|l|}{7} & \multicolumn{3}{|l|}{9} & \multicolumn{6}{|l|}{46} & \multicolumn{3}{|l|}{26} & \\
\hline $\mathrm{N}^{\circ}$ order & \multicolumn{3}{|l|}{3} & \multicolumn{3}{|l|}{4} & \multicolumn{6}{|l|}{8} & \multicolumn{3}{|l|}{7} & \\
\hline
\end{tabular}

Table 1. Presence/absence matrix of crustaceans in lakes of each study province (CA, Continental Antarctic; MA, Maritime Antarctic; SA, Subantarctic islands; SCT, Southern Cool Temperate), based on Pugh et al. $2002^{10}$ and Dartnall et al. $2017^{6}$.

(c) (i) Open Access This article is licensed under a Creative Commons Attribution 4.0 International License, which permits use, sharing, adaptation, distribution and reproduction in any medium or format, as long as you give appropriate credit to the original author(s) and the source, provide a link to the Creative Commons license, and indicate if changes were made. The images or other third party material in this article are included in the article's Creative Commons license, unless indicated otherwise in a credit line to the material. If material is not included in the article's Creative Commons license and your intended use is not permitted by statutory regulation or exceeds the permitted use, you will need to obtain permission directly from the copyright holder. To view a copy of this license, visit http://creativecommons.org/licenses/by/4.0/.

(C) The Author(s) 2020 